\title{
Immersive virtual reality device to support the housing design process
}

\section{SIGRADI2018 TECHNOPOLITICAS \\ xxii congresso da sociedade iberoamericana de gráfica digital 22th conference of the iberoamerican society of digital graphics 07|08|09|novembro|2018 iau usp | são carlos | sp br}

\author{
Marcio Presente de Souza \\ Universidade Estadual de Londrina | Brazil | marciopresente@gmail.com \\ César Imai \\ Universidade Estadual de Londrina | Brazil | cimai@uel.br \\ Maurício Hidemi Azuma \\ Universidade Estadual de Maringá | Brazil | mhazuma@uem.br
}

\begin{abstract}
This paper discusses the process of communication between designers and users during the initial stages of defining the housing design. The objective is to demonstrate the application of a three-dimensional device with virtual reality technology as a tool to facilitate communication between stakeholders in a participatory design context. The method consists of a simulation dynamics, and the application of cognitive walkthrough, verbal protocol and participant observation. The results showed that the simulation model can facilitate the user's understanding of the project represented, as well as stimulate productive discussions about their preferences, needs and wishes about the designed space.
\end{abstract}

Keywords: Simulation; Virtual reality; Participatory design; User.

\section{INTRODUÇÃO}

Este trabalho discute as possibilidades de aplicação da tecnologia de Realidade Virtual (RV) como facilitadora da comunicação entre projetista e usuário durante as etapas iniciais de concepção da arquitetura. As representações bidimensionais do projeto arquitetônico (plantas, cortes, perspectivas), em muitos casos, dificultam a inserção do usuário leigo em uma dinâmica de projeto participativo, uma vez que a linguagem utilizada não faz parte do conhecimento prévio da maioria dos indivíduos.

No entanto, a tecnologia de RV permite uma representação tridimensional de ambientes digitais que aproximam a experiência de visualização do observador de um ambiente real, antes da construção. Essa capacidade de simular $o$ ambiente previamente e apresentá-lo através de uma linguagem de fácil compreensão é uma das características mais relevantes dessa tecnologia, permitindo diversas aplicações no campo da Arquitetura.

Este estudo relata o desenvolvimento de uma ferramenta de RV para facilitar o processo de comunicação entre usuário e projetista nas etapas iniciais do processo de projeto. Além disso, demonstra sua aplicação em um estudo-piloto cujo objetivo foi compreender como essa ferramenta pode facilitar a comunicação de ideias projetuais e coletar preferências do usuário gerando uma dinâmica de processo participativo.

\section{FUNDAMENTAÇÃO TEÓRICA}

Discutir os aspectos relacionados ao processo de projeto em Arquitetura envolve a busca da compreensão do comportamento humano e suas relações com o espaço projetado e edificado. Projetar é uma atividade cuja análise do problema e a busca da solução mais adequada são intrinsicamente relacionadas (Lawson, 2011; Kowaltowski, Moreira, Fabricio \& Petreche, 2011).

Dentre os inúmeros procedimentos conhecidos que suportam o ato de projetar (entrevistas, observações, estudos de caso) destacam-se as avaliações qualitativas, que são capazes de diagnosticar problemas, recomendar modificações e refletir no modo como projetamos os edifícios, ouvindo a opinião dos principais interessados no assunto, os usuários (Ornstein \& Roméro, 1992).

As Avaliações Pré-Projeto (APP), tomam partido do conhecimento técnico e da opinião dos usuários pra nortear os estudos nas etapas iniciais do ato de projetar, coletando dados que orientem o desenvolvimento do projeto e anteveja decisões projetuais equivocadas. $\mathrm{E}$ para que esse objetivo seja alcançado, é necessário um processo de projeto mais participativo, através da comunicação facilitada entre projetista e usuários.

Nas etapas iniciais do processo de projeto arquitetônico, a técnica de simulação dos ambientes projetados mostrase um importante meio para facilitar a comunicação entre os agentes envolvidos. Segundo Voordt e Wegen (2013), as avaliações aliadas à técnica de simulação aprimoram o projeto examinado, melhoram a qualidade do programa de necessidades, da construção e do ambiente construído.

\section{PROCESSO DE PROJETO PARTICIPATIVO}

A qualidade do projeto na arquitetura envolve diferentes dimensões econômicas, sociais e culturais, perpassando os entendimentos e interesses de diferentes agentes: 
projetistas, construtores e usuários, de modo que, um programa de necessidades bem definido influencia consideravelmente a qualidade do projeto (Fabricio, Ornstein \& Melhado, 2010). No caso da habitação, esse conceito de qualidade deve ser, estritamente, uma adequação do espaço circundante às necessidades de seus ocupantes (Imai, 2007).

Voordt e Wegen (2013) apresentam o termo "qualidade funcional", que corresponde à capacidade da edificação de exercer as funções previamente determinadas, oferecendo um nível de apoio às atividades desejadas, retorno econômico favorável e proporção otimizada entre preço e desempenho. Além disso, atribuir qualidade ao projeto significa que o mesmo também possui certa adaptabilidade para abrigar as funções necessárias para diferentes perfis de moradores.

De acordo com Imai (2010), um dos agravantes para se alcançar o entendimento das características e necessidades das pessoas está na relação entre o projetista e o usuário no processo de comunicação, onde, por vezes, a linguagem por meio de instrumentos de representação bidimensional não é compreensível ao leigo.

Para melhorar essa comunicação e o consequente resultado dessas avaliações qualitativas, Kowaltoski, Prata, Pina \& Camargo (2000) recomendam que a utilização da realidade virtual e das simulações de ambientes devem ser aplicadas com mais frequência, não apenas no processo criativo de um projeto novo, mas também dentro do processo de avaliação e coleta de dados para criar um acervo de percepções e pesquisar reações controladas de usuários reais ou potenciais.

O processo de projeto participativo é essencialmente uma pesquisa com métodos e técnicas voltados para construção interativa de conhecimento prático ou tácito, sendo que, na arquitetura, essa prática apresenta potencial relevante por conseguir dar voz aos vários agentes envolvidos no desenvolvimento de um projeto, e fomentar uma discussão mais consistente sobre as decisões projetuais com base nas informações oriundas da diversidade das fontes de informação (Spinuzzi, 2005).

\section{SIMULAÇÕES EM REALIDADE VIRTUAL}

As técnicas de simulações podem ser utilizadas como um experimento virtual, um modelo capaz de fazer projeções de eventos futuros, inserindo-se tanto no contexto de descoberta quanto no contexto da prova (Vicente, 2005). A simulação é a representação do comportamento ou características de um sistema através do uso de outro sistema, especialmente um programa de computador projetado para o propósito, sendo que a combinação entre o computador e o homem favorece a criação de cenários, que são novas formas de se imaginar a realidade, testar hipóteses e aprender interativamente (Wang, 2013; Jerald, 2016).

Por meio da realidade virtual, as simulações podem representar ambientes digitais muito semelhantes aos ambientes reais. A RV compreende um ambiente digital avançado, com múltiplas interfaces, onde o usuário pode navegar e interagir, em tempo real, em espaços tridimensionais gerados por computador, usando dispositivos multissensoriais (Kirner \& Kirner, 2011; Jerald, 2016). Essa tecnologia é capaz de produzir um espaço digital no qual os movimentos do usuário são rastreados e seus arredores são renderizados (compostos digitalmente), podendo contribuir com o processo de comunicação entre usuários e projetistas, para aferir, previamente, o grau de satisfação dos ocupantes com a sua habitação (Malard, Conti, Souza \& Campomori, 2002; Fox, Arena \& Bailenson, 2009).

A RV pode ser classificada como não imersiva, visualizada no modo janela, quando o utilizador interage com o ambiente através de imagens apresentadas em um monitor. E imersiva, quando a interação acontece pelo uso de dispositivos multissensoriais como capacetes, salas de multiprojeção, luvas de dados ou óculos 3D (Kirner \& Kirner, 2011). Na característica imersiva, ao perder o contato com o ambiente real, o usuário simula a sensação de estar presente no ambiente virtual, e através da interação, consegue explorar diversas possibilidades de visualização do projeto.

Um dos dispositivos comumente utilizados para RV consiste em um Head-Mounted Display (HMD), comercialmente conhecido como óculos de Realidade Virtual ou óculos 3D. A simulação do projeto por meio dos óculos 3D é capaz de apresentar um panorama de imagens 360 graus, melhorando a maneira de interação do usuário em sistemas de localização e permite acessar ampla variedade de informação visual em $R V$, que pode ser estudado por meio da experiência do usuário (See \& Cheok, 2015).

Segundo Higuera-Trujillo, Maldonado \& Millán, (2017) panoramas 360 graus oferecem respostas psicológicas (percepções sobre os ambientes simulados) mais próximas do espaço construído quando comparados a fotografias convencionais e cenários em RV não imersiva. Esse efeito é obtido, principalmente pela capacidade desse tipo de representação de proporcionar ao usuário o senso de presença (sensação de estar em um espaço enquanto o seu corpo se encontra em outro local), a experiência visual e a interação com os ambientes simulados (Higuera-Trujillo, Maldonado \& Millán, 2017).

A forma de validação dessa tecnologia consiste na análise da experiência do usuário (User Experience), que é um meio de avaliar a usabilidade de interfaces computacionais. Sua definição surge dos trabalhos interligados a percepção, ação, motivação, emoção e cognição do usuário com sistemas simulados (Hassenzahl, 2010). Para estudar o sistema sob o ponto de vista do usuário, o dispositivo de simulação é tratado como um produto que precisa ser utilizável, demostrando usabilidade satisfatória. Ao desenvolver sistemas que analisem a experiência do usuário no cumprimento de tarefas através da interação, a usabilidade pode ser avaliada (Kuliga, Thrash, Dalton \& Holscher, 2015; Madathil \& Greenstein, 2017).

No estudo da RV, a interação do usuário com o sistema projetado é fundamental. A interação é o modo como o usuário se comunica com a interface digital, principalmente através de dispositivos (óculos, luvas, capacetes), sendo que essa comunicação pode ser apenas facilitadora da visualização dos objetos 
representados ou ainda, permitir a realização de tarefas específicas (Bowman, Kruijff, Laviola Jr. \& Poupyrev, 2004).

A partir da interação do usuário com os sistemas simulados, o feedback é um artifício essencial, pois pode extrair do usuário a informação decorrente de uma ação do mesmo ou ainda, comunicar o usuário de uma ação do próprio dispositivo. De modo que o feedback comunica ao usuário os resultados de uma ação ou tarefa, ajudando a entender o estado de interação do sistema e a conduzir ações futuras (Jerald, 2016).

\section{MATERIAIS E MÉTODOS}

O método principal utilizado nesse estudo consiste em uma dinâmica de simulação estruturada por meio de um roteiro de aplicação da pesquisa. Este roteiro contém as orientações da ordem dos procedimentos a serem executados pelo avaliador, assim como as questões que devem ser respondidas pelo usuário. As questões estimulam o usuário a propor alterações no projeto, verificam a aceitação de determinada solução projetual e aspectos dimensionais como tamanho dos ambientes, das circulações e das aberturas, relacionando com suas necessidades de uso.

A estrutura do roteiro possui, em sua maioria, questões com cinco níveis de gradação - Péssimo, Ruim, Regular, Bom e Excelente - para facilitar a interpretação dos resultados. Além disso, possui questões abertas que estimulam o usuário a propor modificações dimensionais ou de escolha de materiais do projeto e servem ainda, para que o usuário avalie a dinâmica de simulação e comente sobre a experiência de uso do dispositivo utilizado na simulação.

Algumas técnicas foram associadas à simulação: o percurso cognitivo, que consiste em percorrer os ambientes simulados realizando tarefas pré-definidas; o protocolo verbal, que estimula o usuário a explicar em voz alta aquilo que está pensando, comentando suas impressões pessoais sobre o sistema enquanto realiza a tarefa; e a observação do participante, que consiste na captação por parte do pesquisador, do comportamento do usuário ao utilizar o sistema (Smythe \& Spinillo, 2017).

O método foi validado por meio de um estudo-piloto realizado com seis pessoas de perfis diversificados, com variação de gênero, idades e graus de instrução, realizado em uma sala de aula de um estabelecimento de ensino. Essa primeira etapa foi realizada para reduzir riscos ou incertezas no método que se pretende utilizar, ajudando a desenvolver estratégias mais adequadas para mitigação de riscos, caso o método venha a falhar (Turner, 2005). Um estudo-piloto permite uma investigação ampla das possibilidades de pesquisa, e caracteriza-se como um ensaio que busca experimentar, analisar, reformular e aperfeiçoar as ferramentas em questão (Turner, 2005).

O objetivo deste primeiro estudo foi explorar diferentes estratégias de abordagem junto ao usuário, verificar a viabilidade da configuração do cenário de simulação, a dinâmica proposta, e os recursos necessários para coletar e analisar os dados.

\section{DISPOSITIVO TRIDIMENSIONAL EM RV IMERSIVA}

O fundamento principal do dispositivo proposto consiste na reprodução de um projeto habitacional em um ambiente virtual imersivo. $O$ projeto representado foi vencedor de um Concurso Nacional de Arquitetura realizado pela Companhia de Desenvolvimento Habitacional do Distrito Federal (CODHAB/DF) no ano de 2016 para a Região Administrativa de Samambaia. A escolha desse projeto parte do princípio de que o mesmo passou pelo crivo de uma equipe técnica e foi elencado como o mais qualificado para o concurso em questão.

Para desenvolver o sistema de RV no dispositivo, constatou-se que um procedimento de tour virtual poderia contribuir para a dinâmica proposta, sendo constituído pela conexão de uma série de imagens 360 graus, produzindo uma espécie de percurso onde o usuário pudesse receber as informações deslocando-se pelos ambientes. $O$ uso de imagens 360 graus nesse tipo de representação permite a visualização em 6 Graus de Liberdade (GLB), efeito que proporciona a imersão total no ambiente virtual.

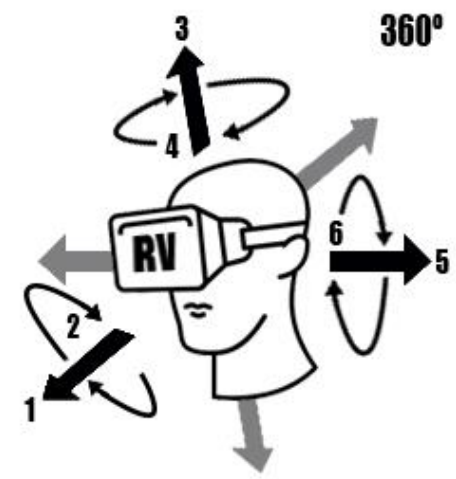

Figura 1: 6GLB: Mover p/ frente e atrás (1 e 2); Acima e Abaixo (3 e 4); De um lado para o outro (5 e 6). Fonte: Dos autores.

O projeto escolhido foi modelado no programa $3 D$ Studio Max, e posteriormente foram produzidos panoramas de cada ambiente, que consistem em imagens renderizadas (compostas digitalmente) de múltiplos ângulos de visão (6GLB), que são combinadas em um formato esférico. Para a produção das imagens panorâmicas foi utilizado o plugin V-Ray 3.0.

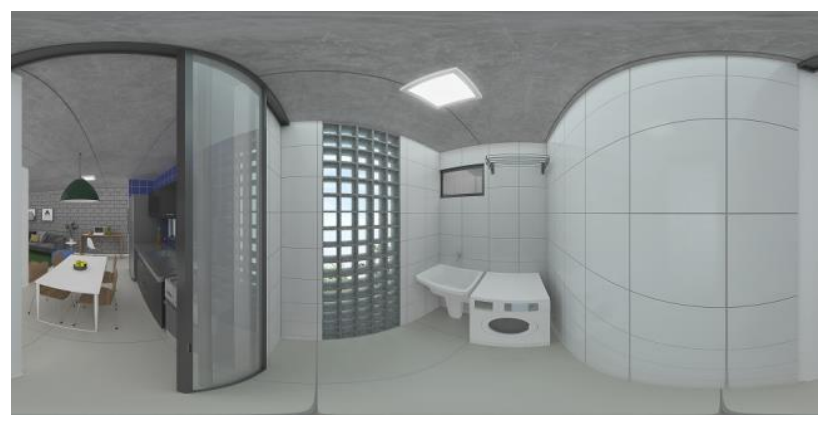

Figura 2: Imagem esférica renderizada. Fonte: Dos autores.

Após a produção das imagens esféricas de cada ambiente, foram testadas três plataformas para 0 processamento e produção do tour virtual: PANOTour, SentioVR e ImersioVR. A plataforma ImersioVR foi 
escolhida pois possui uma interface mais simples de utilização e possibilita a produção de até 20 imagens por tour de forma gratuita. Por meio dessa interface são configurados hotspots para interação. Estes constituem uma espécie de marcador inserido na imagem com a finalidade de direcionar o usuário até a imagem seguinte, ou retornar a imagem inicial. Dois tipos de hotspots podem ser configurados, um ícone posicionado em qualquer ponto da imagem, ou setas de avançar ou retroceder que ficam flutuando no centro das imagens visualizadas.

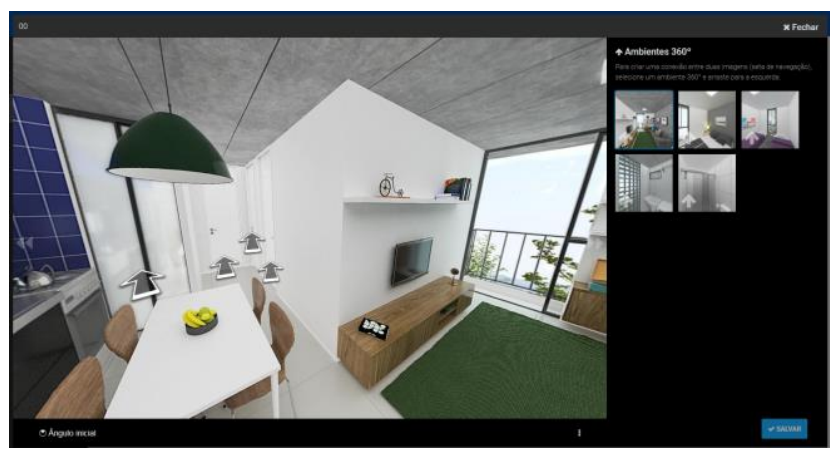

Figura 3: Interface de edição do tour virtual na plataforma ImersioVR. Fonte: Dos autores.

Após a configuração do tour, a plataforma gera um link que direciona para a visualização das imagens no site da mesma. Dessa forma, o link pode ser acessado por um smartphone inserido nos óculos 3D para realização da simulação. Os procedimentos para a realização da simulação no dispositivo consistem em: (1) acessar o link gerado na plataforma ImersioVR através do smartphone; (2) ativar o modo cardboard na visualização das imagens (a imagem é subdividida e relocada no formato paisagem); (3) inserir o smartphone nos óculos 3D; (4) colocar os óculos na cabeça e ajustar o foco das imagens através dos botões de ajuste localizados na parte superior dos óculos.

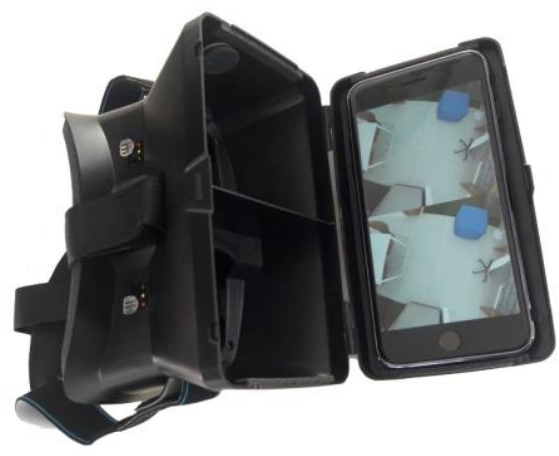

Figura 4: Óculos 3D com smartphone. Fonte: Dos autores.

Tais botões de ajuste de foco dos óculos calculam a distância entre os olhos do usuário, sendo necessário o ajuste dessa distância por cada participante no início da simulação. Os óculos 3D apresentam também uma alça para fixação na cabeça do participante, de modo que ele não precise segurá-los e tenha a total liberdade de movimentação da cabeça (6GLB). O giroscópio do smartphone identifica os movimentos do usuário no espaço e sincroniza sua posição de acordo com a imagem que está sendo visualizada na tela, reforçando a sensação de imersão.

\section{RESULTADOS}

As primeiras questões do roteiro objetivaram identificar o perfil dos participantes: gênero, idade, estado civil, número de moradores e tipo de residência. $\mathrm{O}$ grupo de seis participantes foi composto, em sua maioria, pelo gênero feminino (4), com faixa-etária de 20 a 36 anos, a maioria solteiros e sem filhos (4) residindo em apartamentos (4). Após esta entrevista prévia, o projeto escolhido foi apresentado aos participantes da dinâmica por meio dos óculos 3D e, em seguida foi realizado o tour virtual pelos ambientes. A tarefa consistiu em identificar corretamente os ambientes do projeto para, em seguida, serem questionados sobre aspectos perceptivos em relação ao espaço simulado.

Durante o tour os participantes foram reconhecendo elementos que caracterizam o projeto (percurso cognitivo) e expressando suas impressões, dúvidas ou problemas encontrados (protocolo verbal). A maioria identificou todos os ambientes corretamente, além de descrever espaços secundários como entrada social, mini homeoffice e sacada. Esse fato demostra o potencial relevante da ferramenta de apresentar aos indivíduos, detalhes menores do projeto.

A primeira questão do roteiro indagou o participante a identificar a tipologia da edificação através do dispositivo de RV, tendo as opções de casa térrea, sobrado, apartamento ou outro. A maioria identificou corretamente o apartamento simulado, principalmente pelo tamanho reduzido dos ambientes, janela do banheiro com saída pra lavanderia e a visão do entorno da edificação através da representação das janelas.

Logo após, eles foram questionados sobre a percepção em relação ao tamanho dos ambientes representados. De maneira geral, sala/cozinha e quartos foram apontados como mais compreensíveis em relação ao espaço projetado, enquanto que banheiro e lavanderia constatouse certa dificuldade de compreensão do tamanho dos espaços. Esse resultado demonstra uma limitação de representação do dispositivo utilizado, sendo que, nos ambientes muito pequenos, ocorre uma distorção da perspectiva das imagens panorâmicas quando 0 participante olha para baixo, ocasionando dificuldades de compreensão de alguns aspectos do projeto.
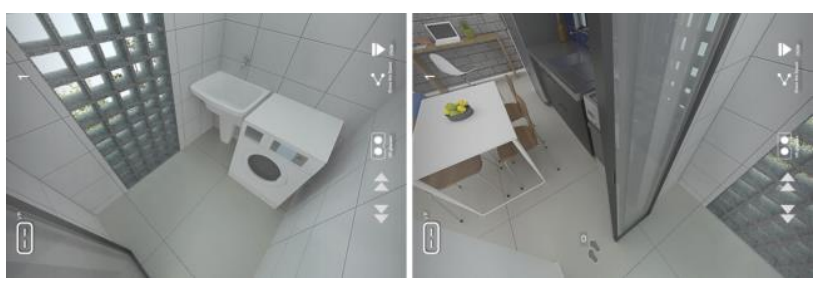

Figura 5: Distorção da perspectiva. Fonte: Dos autores.

Com relação aos estímulos para a modificação do projeto, - dispositivo possibilitou que os participantes associassem 0 espaço representado as suas necessidades de uso, apontando modificações ou preferências que seriam mais adequadas ao seu perfil. Surgiram sugestões desde ampliação da cozinha, 
mudança na posição da porta de entrada, inversão de leiaute dos móveis ou ainda mudança nos revestimentos utilizados.

A representação dos materiais nesse dispositivo virtual também foi um ponto importante identificado no estudo. Os participantes tendem a compreender bem o emprego dos revestimentos, móveis e objetos, pela característica imersiva de representação do espaço, podendo visualizar em diferentes ângulos o mesmo ambiente. Esse efeito foi identificado pelos comentários durante a experiência de simulação, sendo que a maioria apontou compreender bem os espaços representados dessa maneira, semelhante a uma caminhada dentro do ambiente, próximo à realidade, enxergando melhor os detalhes.

Quanto à avaliação dos participantes sobre o nível de dificuldade na utilização do dispositivo, foram apontados aspectos de fácil utilização. Pela observação da dinâmica foi possível constatar que após a explicação do funcionamento do mecanismo e ajustes nos óculos, todos utilizaram o sistema sem grandes dificuldades ou desconforto. Foi identificado que o dispositivo de RV melhora a compreensão do usuário sob aspectos específicos do ambiente simulado, como leiaute, objetos, revestimentos, aberturas e fechamentos. No entanto, a ferramenta utilizada não possui a capacidade de representar a totalidade do projeto, mostrando todos os ambientes simultaneamente.

Em uma representação bidimensional como uma planta baixa, o usuário compreende a relação entre os ambientes e aspectos dimensionais, como a área total do projeto. No entanto, este dispositivo utilizado possui limitações para representar esses aspectos, principalmente pela característica de apresentação das imagens dos ambientes separados. A tabela a seguir mostra as características principais das informações coletadas.
Tabela 1: Síntese da contribuição da dinâmica de simulação no processo de projeto participativo. Fonte: Dos autores.

\begin{tabular}{|c|l|}
\hline $\begin{array}{c}\text { Capturou mais } \\
\text { informações }\end{array}$ & $\begin{array}{l}\text { Preferências de uso dos espaços, } \\
\text { alterações de leiaute e questões } \\
\text { relacionadas ao mobiliário, revestimentos } \\
\text { e iluminação. }\end{array}$ \\
\hline $\begin{array}{c}\text { Capturou } \\
\text { menos } \\
\text { informações }\end{array}$ & $\begin{array}{l}\text { Sobre mudanças da posição dos } \\
\text { ambientes na planta baixa. Também } \\
\text { coletou menos informações sobre a } \\
\text { dimensão dos espaços e circulações. }\end{array}$ \\
\hline $\begin{array}{c}\text { Não capturou } \\
\text { adequadamente } \\
\text { as informações }\end{array}$ & $\begin{array}{l}\text { Sobre a posição dos ambientes projetados } \\
\text { e sua relação com os demais espaços. } \\
\text { Dificuldade de entender as circulações } \\
\text { entre os ambientes, a origem e destino } \\
\text { das aberturas e o conjunto do projeto. }\end{array}$ \\
\hline $\begin{array}{c}\text { O que mais } \\
\text { contribui no } \\
\text { processo? }\end{array}$ & $\begin{array}{l}\text { Estimula o usuário a sentir-se dentro do } \\
\text { espaço e identificar inconsistências na } \\
\text { finalidade do ambiente projetado para seu } \\
\text { uso, antevendo conflitos projetuais. }\end{array}$ \\
\hline $\begin{array}{c}\text { O que menos } \\
\text { contribui no } \\
\text { processo? }\end{array}$ & $\begin{array}{l}\text { Percepção em relação ao tamanho dos } \\
\text { ambientes e a totalidade do projeto. O } \\
\text { usuário tem dificuldadle de compreender a } \\
\text { área total da habitação. }\end{array}$ \\
\hline $\begin{array}{c}\text { Qual } \\
\text { informação não } \\
\text { contribui? }\end{array}$ & $\begin{array}{l}\text { Aspectos funcionais que dependem da } \\
\text { compreensão do conjunto do projeto não } \\
\text { são captados por esse instrumento, como } \\
\text { o funcionamento das circulações e relação } \\
\text { com os demais ambientes. }\end{array}$ \\
\hline
\end{tabular}

\section{CONCLUSÃO}

O estudo buscou explorar a potencialidade de aplicação de um dispositivo de simulação em RV nas etapas iniciais do processo de projeto, cujo objetivo principal era facilitar a comunicação de ideias dos agentes envolvidos e levantar preferências espaciais de cada usuário ouvido. Nota-se, que no ambiente virtual, a sensação de imersão proporciona uma experiência de vivenciar o espaço

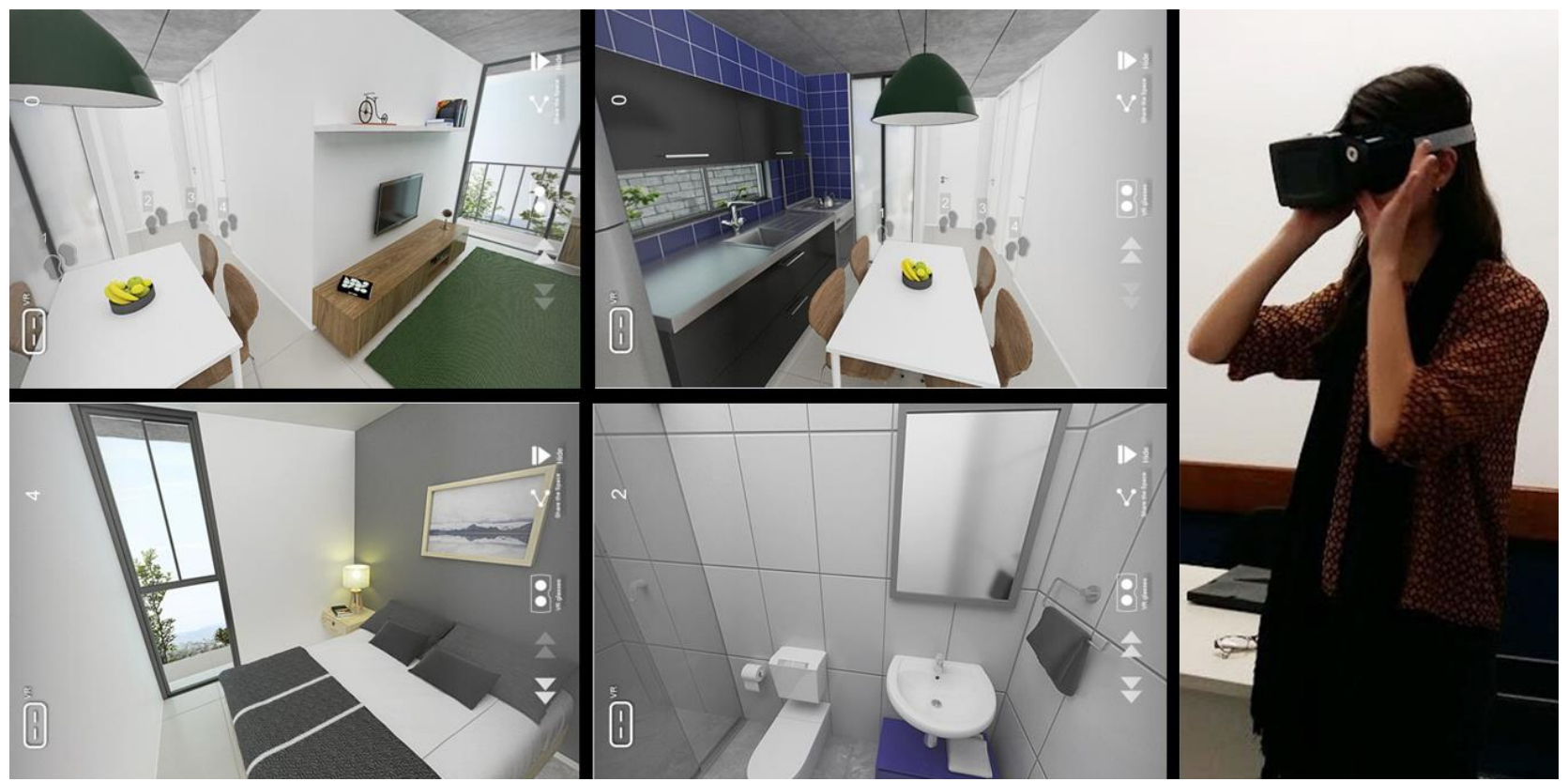

Figura 6: Perspectivas dos ambientes simulados no dispositivo de RV e usuário durante a dinâmica. Fonte: Dos autores. 
projetado no período anterior à construção da edificação, levando a uma avaliação das soluções por parte dos usuários, possibilitando antever conflitos projetuais relacionados às suas necessidades de uso dos ambientes. A capacidade da ferramenta em permitir a antevisão sobre o aceite ou não das soluções projetuais possui grande potencial para aplicação em avaliações prévias do projeto, seja na programação arquitetônica ou na Avaliação Pré-Projeto.

Diferentemente das avaliações técnicas mais tradicionais, a inserção das simulações insere o usuário de um modo mais dinâmico no processo de projeto, mais participativo. A estrutura do método desenvolvido neste estudo buscou estimular justamente essa participação mais ativa do usuário no processo. E diferente do que ocorre com questionários/entrevistas convencionais, na dinâmica proposta o usuário precisa movimentar-se, interagir com o sistema e expressar, de modo mais crítico, sua opinião durante todo o processo, melhorando a qualidade da informação coletada.

É importante destacar que a interação é um ponto fundamental para que a simulação produza resultados satisfatórios. Desenvolver métodos e técnicas de ampliação da interação do usuário com o dispositivo de RV seria um caminho interessante para pesquisas futuras. A interação ativa a percepção dos usuários permitindo um processo mais fluido e uma liberdade maior para o mesmo compreender o espaço projetado e expressar ao avaliador o seu ponto de vista. Em se tratando de avaliações com usuários, cujas informações são influenciáveis por inúmeras variáveis, faz-se necessário o uso de multi métodos de abordagem. Ao utilizar diferentes técnicas neste estudo, é possível coletar uma quantidade maior de informação sobre o usuário e sua compreensão do projeto, e processá-los de maneira mais eficiente, ainda que esses dados apresentem características subjetivas. Cada método coleta dados de diferentes origens e a correlação entre eles ajuda na interpretação da informação pelo pesquisador.

\section{AGRADECIMENTOS}

Os autores agradecem a CAPES e Fundação Araucária.

\section{REFERÊNCIAS}

Bowman, D. A., Kruijff, E., Laviola Jr., J. J., \& Poupyrev, I. (2004). 3D User interfaces: Theory and practice. AddisonWesley.

Fabricio, M. M., Ornstein, S. W., \& Melhado, S. B. (2010). Conceitos de qualidade no projeto de edifícios. In: M. M. Fabricio, \& S. W. Ornstein, Qualidade no projeto de edifícios (pp. 5-22). São Carlos: RiMa Editora.

Fox, J., Arena, D., \& Bailenson, J. N. (2009). Virtual reality: a survival guide for the social scientist. Journal of Media Psychology, 21(3), 95-113. doi.org/10.1027/18641105.21.3.95

Hassenzahl, M. (2010). Experience Design: Technology for all the right reasons. Synthesis Lectures on Human-Centered Informatics, 3(1), 1-95. doi.org/10.2200/S00261ED1V01Y201003HCI008

Higuera-Trujillo, J. L., Maldonado, J. L., \& Millán, C. L. (2017). Psychological and physiological human responses to simulated and real environments: A comparison between photographs, $360^{\circ}$ panoramas, and virtual reality. Applied Ergonomics, 398-409. doi.org/10.1016/j.apergo.2017.05.006

Imai, C. (2007). A utilização de modelos tridimensionais físicos em projetos de habitação social: o Projeto Casa Fácil. 326f. Tese (Doutorado) Programa de Pós-Graduação em Arquitetura e Urbanismo, Universidade de São Paulo, São Paulo.

Imai, C. (2010). O sonho da moradia no projeto: o uso da maquete arquitetônica na simulação da habitação social. Maringá: EdUEM.

Jerald, J. (2016). The VR Book: Human-Centered Design for Virtual Reality. ACM Books.

Kirner, C., \& Kirner, T. G. (2011). Development of an educational spatial game using an augmented reality authoring tool. Journal of Computer Information Systems and Industrial Management Applications, 3, 602-611.

Kowaltowski, D. C., Moreira, D. C., Fabricio, M. M., \& Petreche J. R. D. (2011). O Processo de projeto em arquitetura: da teoria à tecnologia. São Paulo: Oficina de textos.

Kowaltowski, D. C., Prata, A. R., Pina, S. A., \& Camargo, R. F. (2000). Ambiente construído e comportamento humano: necessidade de uma metodologia. In: ENTAC 2000, Encontro Nacional de Tecnologia no Ambiente Construído, Anais... 26-28 de abril, Salvador.

Kuliga, S. F., Thrash, T., Dalton, R. C., \& Holscher, C. (2015). Virtual reality as an empirical research tool - Exploring user experience in a real building and a corresponding virtual model. Computers, Environment and Urban Systems, 54, 363-375. doi.org/10.1016/j.compenvurbsys.2015.09.006

Lawson, B. (2011). Como arquitetos e designers pensam. São Paulo: Oficina de textos.

Madathil, K. C., \& Greenstein, J. S. (2017). An investigation of the efficacy of collaborative virtual reality systems for moderated remote usability testing. Applied Ergonomics, 65, 501-514. doi.org/10.1016/j.apergo.2017.02.011

Malard, M. L., Conti, A., Souza, R. C., \& Campomori, M. J. (2002). Avaliação pós-ocupação, participação do usuário e melhoria da qualidade de projetos habitacionais: uma abordagem fenomenológica com o apoio do Estado. In A. K. Abiko, \& S. W. Ornstein, Inserção urbana e avaliação pósocupação (APO) da habitação de interesse social. São Paulo: FAUUSP - Coletânea Habitare/FINEP.

Ornstein, S. W., \& Roméro, M. (1992). Avaliação Pós-Ocupação (APO) do ambiente construído. São Paulo: Studio Nobel, Editora da Universidade de São Paulo.

See, Z. S., \& Cheok, A. D. (2015). Virtual reality 360 interactive panorama reproduction obstacles and issues. Virtual Reality, 19(2). doi.org/10.1007/s10055-014-0258-9

Smythe, K. C., \& Spinillo, C. G. (2017). Avaliação de métodos e técnicas para inserção do usuário na fase inicial do processo de design de sistemas wayfinding. Infodesign, 17(1), 14-29.

Spinuzzi, C. (2005). The methodology of participatory design. Technical Communication, 52(2), 163-174.

Turner, J. R. (2005). The Role of pilot studies in reducing risk on project and programmes. International Journal of Project Management, 23, 1-6.

Vicente, P. (2005). O uso de simulação como metodologia de pesquisa em ciências sociais. Cadernos EBAPE.BR, 3(1),19.

Voordt, T. J., \& Wegen, H. B. (2013). Arquitetura sob o olhar do usuário: programa de necessidades, projeto e avaliação de edificações. São Paulo: Oficina de Textos.

Wang, D. Simulation Research. (2013). In L. Groat, \& D. Wang. Architectural Research Methods. Second Edition. New York: John Wiley \& Sons. 